\title{
Gastric and Peritoneal Involvement of Human Herpes Virus Type 8 Related Kaposi Sarcoma in a Patient with Acquired Immunodeficiency Syndrome
}

\author{
Nuno Ribeiro Ferreira, Patrícia Vicente, Rui Costa, Carolina Gouveia, Sofia Mateus \\ Internal Medicine Department, Hospital São Francisco Xavier, Centro Hospitalar Lisboa Ocidental, Lisboa, Portugal
}

Doi: 10.12890/2015_000260 - European Journal of Case Reports in Internal Medicine - ๑ EFIM 2015

Received: 30/07/2015

Accepted: 11/08/2015

Published: 04/09/2015

How to cite this article: Ribeiro Ferreira N, Vicente P, Costa R, Gouveia C, Mateus S. Gastric and peritoneal involvement of human herpes virus 8 related Kaposi sarcoma in a patient with acquired immunodeficiency syndrome. EJCRIM 2015;2:doi: 10.12890/2015_000260

Conflicts of Interests: The authors declare that they have no competing interests.

Patient's Consent: The authors declare they obtained consent for publication from a relative.

Acknowledgements: Authors would like to thank Emilia Valadas, Ana Claudia Miranda and Fátima Grenho for their contribution to the final revision of the manuscript.

This article is licensed under a Commons Attribution Non-Commercial 4.0 License

\section{ABSTRACT}

Kaposi's sarcoma (KS) is one of the most frequent neoplastic diseases in patients infected with human immunodeficiency virus (HIV). The authors report the case of a 40-year-old male with ascites, peripheral edema and peritoneal carcinomatosis secondary to a gastric KS related to human herpes virus type 8 (HHV-8). The patient had severe immunodeficiency, with a TCD4+ count of 86 cells/ $\mu$ land newly diagnosed acquired immunodeficiency syndrome. His clinical condition rapidly deteriorated, with multiorgan failure, and he died without the possibility of initiating antiretroviral therapy or chemotherapy. To the authors' knowledge, carcinomatosis is a rare feature in KS.

\section{LEARNING POINTS}

- In a patient with acquired immunodeficiency syndrome (AIDS) presenting with Kaposi sarcoma (KS) with skin involvement, the possibility of visceral disease must be considered, even if asymptomatic.

- Multicentric Castleman disease (MCD) and primary effusion lymphoma must be considered in every HIV-infected patient with anasarca, poliadenopathy and hypoalbuminemia.

- KS may present in a disseminated form and gastrointestinal involvement may led to ascites and carcinomatosis.

\section{KEYWORDS}

Human immunodeficiency virus, acquired immunodeficiency syndrome, human herpes virus 8, Kaposi sarcoma, carcinomatosis.

\section{INTRODUCTION}

Kaposi's sarcoma (KS) results from the abnormal proliferation of endothelial pluripotent cells. Skin involvement is frequently observed, though lungs, gastrointestinal tract and lymph nodes may also be affected. Visceral involvement of KS is frequently asymptomatic and found accidently on imaging studies. Disseminated KS is associated with poor prognosis. In the gastrointestinal tract, KS may affect the small intestine, stomach, colon and rectum, but extension to peritoneum is not a frequent feature ${ }^{[1]}$.

We report a case of a human herpes virus type 8 (HHV-8)-related gastric KS, disseminated to skin and peritoneum, with an unusual clinical presentation both in extension and severity, in a patient with newly diagnosed acquired immunodeficiency syndrome (AIDS).

\section{CASE REPORT}

A 40-year-old heterosexual male presented to the emergency department with complaints of asthenia, fatigue, 20-pound weight loss 
over the past 15 months, and an increase in abdominal volume and peripheral edema in the previous 4 weeks. His medical history was unremarkable, except for episodes of unprotected sexual intercourse with commercial sex workers in the past.

Physical examination revealed a cachectic patient with prominent ascites and diffuse painless nodularity, with no signs of portal hypertension. His vital signs were normal. He had a soft edema in both legs, extending to the inguinal area and scrotum. A skin examination showed three well-defined violaceous non tender plaques, with $2-3 \mathrm{~cm}$ on both feet (Fig. 1). No mucosal lesions were found and no enlarged lymph nodes were palpable. Cardiopulmonary examination was normal.
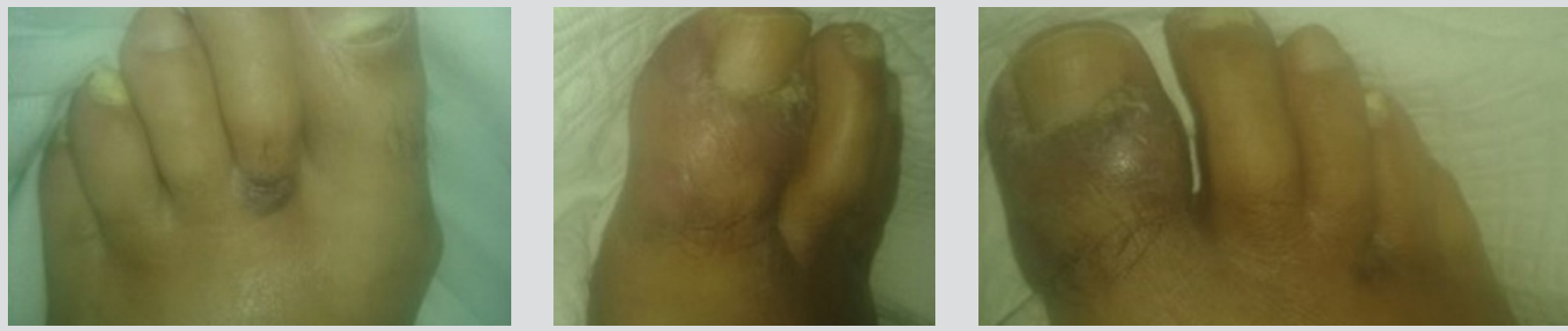

Figure 1: Kaposi sarcoma lesions.
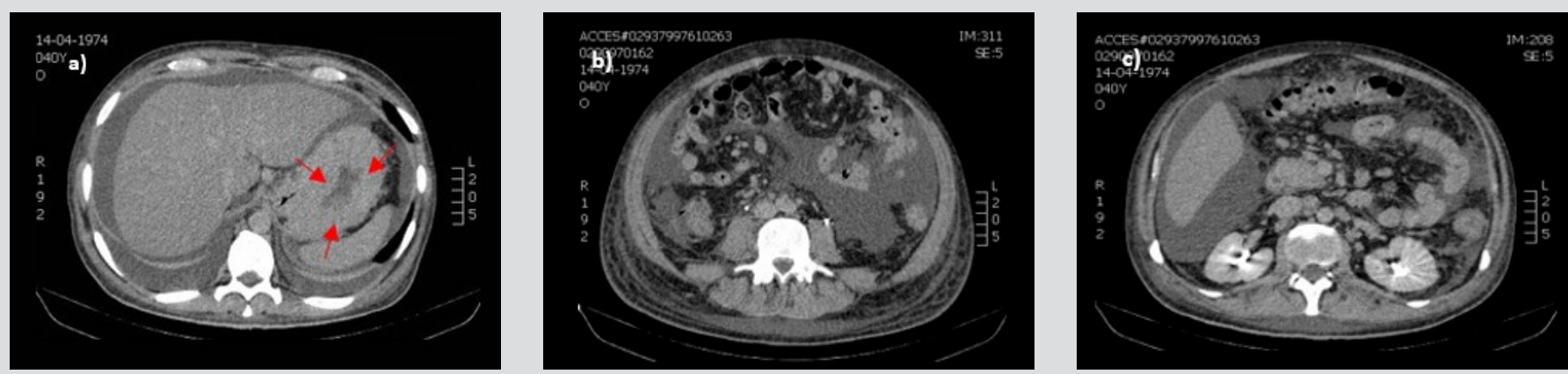

Figure 2: Abdominal CT-scan demonstrating (a) a marked thickness in gastric wall, (b) massive ascitis and (c) multiple intra-abdominal adenopathies.

The patient was admitted to hospital with wasting syndrome with anasarca, and we suspected incidence of an abdominal neoplastic disease. Routine laboratory investigation was carried out, and complete blood count was found to be within normal range, including haemoglobin level and platelet count. A mild elevation in gama-glutamil transpeptidase and alkaline phosphatase (222 U/L and $205 \mathrm{U} / \mathrm{L}$, respectively) was noted, while lactate dehydrogenase levels were normal. Albumin levels were $1.3 \mathrm{~g} / \mathrm{dl}$. Due to wasting syndrome, violaceous skin lesions and risk factors, a viral screening was ordered and was negative for both hepatitis B and C, but positive for human immunodeficiency virus type 1 (HIV-1). Flow cytometric analysis revealed a lymphocyte count of $2945 \mathrm{cells} / \mu \mathrm{l}$; severe depletion of TCD4+ cell ( $86 \mathrm{cells} / \mu \mathrm{l})$ and high HIV-1 plasma replication (952.198 copies/ml). Protein electrophoresis revealed hypoalbuminemia, while immunoelectrophoresis was normal. A thoracic and abdominal computerized tomography scan showed a marked thickness in the gastric wall, multiple adenopathies, massive ascites with peritoneal implants and carcinomatosis (Fig. 2). No parenchymal lesions where found in the lungs. An esophagogastroduodenoscopy was performed, revealing hypertrophic, thickened and violaceous folds in the gastric wall (Fig. 3).

Gastric biopsies identified hyperplasic and ulcerated mucosa, with an intense inflammatory infiltrate. Proliferation of spindle cells forming poorly defined vascular spaces with extravasated erythrocytes were found in the lamina propria. Immunohistochemistry showed endothelial and glandular cells positively stained for HHV-8 and cytomegalovirus (CMV) with no evidence of carcinoma (Fig. 4).

A diagnostic paracentesis was performed and analysis of the ascitic fluid revealed a non-purulent fluid with 350 cells/ $\mu \mathrm{L}$. Protein and albumin levels were low $(0.3 \mathrm{~g} / \mathrm{dL}$ and $0.07 \mathrm{~g} / \mathrm{dl}$, respectively). Glucose, lactate dehydrogenase and adenosine deaminase levels were normal. Microbiological cultures were negative, including for mycobacteria. Microscopic observation identified medium-sized cells with raised nucleus/cytoplasm relation and basophilic cytoplasm. Immunocytometry showed $82.3 \%$ lymphocytes with no phenotypic abnormalities, and immunohistochemistry revealed reactivity for CD31+ and CD34+ in ascitic fluid cells compatible with KS deposits.

The patient was considered for antiretroviral therapy and systemic chemotherapy, but extreme cachexia, disseminated KS and suspected active opportunistic infection with HHV-8 discouraged this immediate approach. A lymph node biopsy was considered in order to exclude 


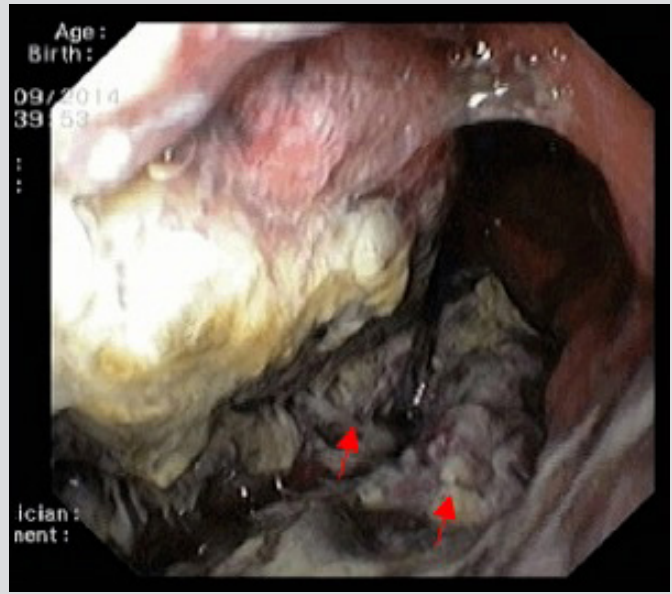

Figure 3: Esophagogastroduodenoscopy revealing soft, hypertrophic, thickened and violaceous folds in the gastric wall.

multicentric Castleman disease (MCD), but there was a sudden clinical deterioration, one week after admission, with multiorgan failure, hypotension and fever. A septic shock was suspected and immediate broad spectrum antibiotics and vasopressor support were initiated but the clinical condition evolved to a refractory shock and death within 72 hours.

Post-mortem immunofluorescence results revealed high titers of immunoglobulin G against HHV-8 (1:64) and a serum sample was positive for HHV-8 deoxyribonucleic acid (DNA) by protease chain reaction amplification of a 111pb fragment from the OR 26 region of the virus. HHV-8 viral load was 23467 copies per ml (post-mortem result).

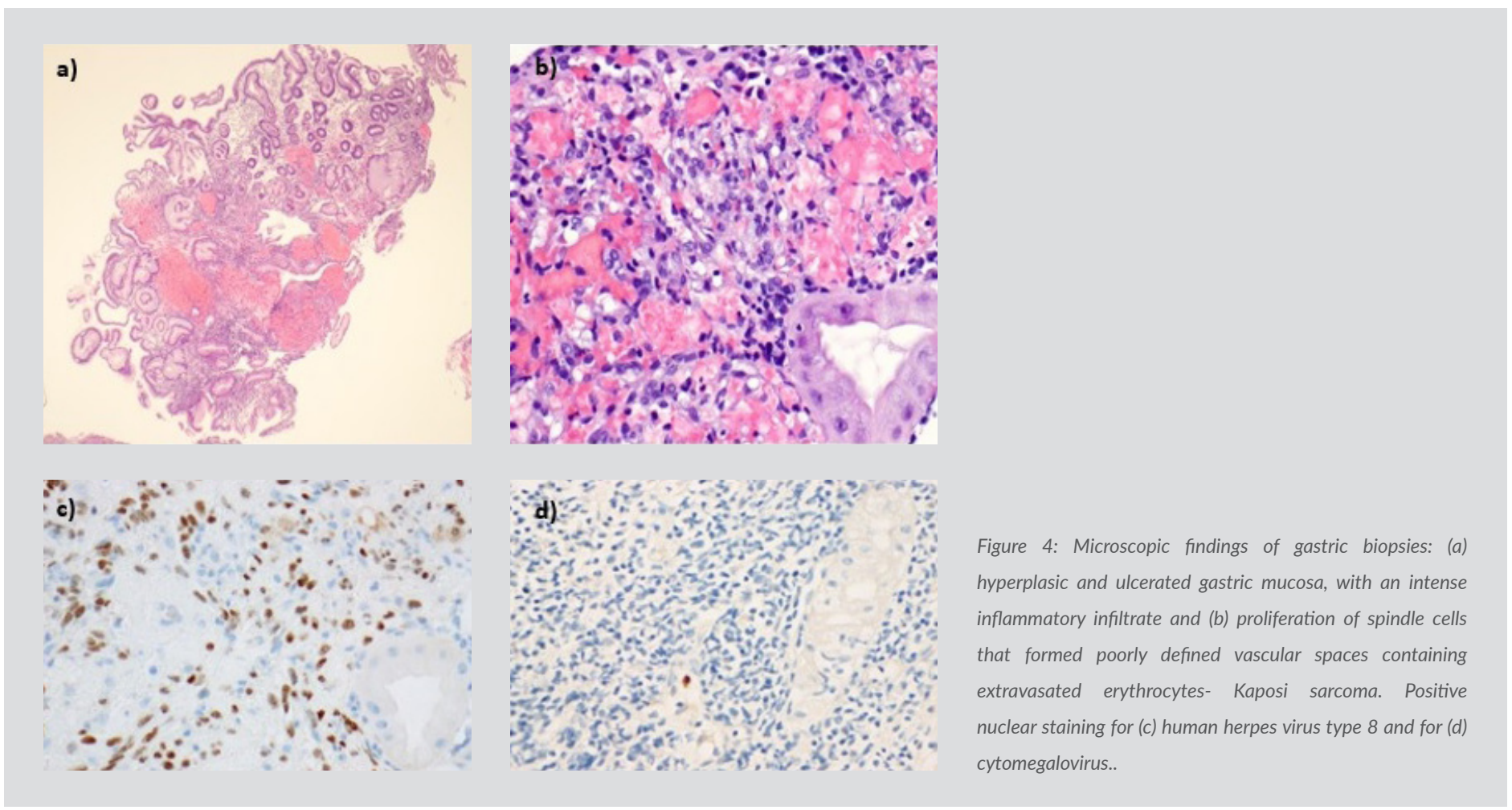

\section{DISCUSSION}

In advanced HIV-infection, KS may have a muco-cutaneous disseminated distribution and affect gastrointestinal organs. However, peritoneal carcinomatosis is a rare feature and MCD and primary effusion lymphoma (PEL) must be considered in these cases ${ }^{[2]}$. In the case of this patient, immunohistochemistry analysis identified KS cell deposits and the widespread intrabdominal extension provides an explanation 
for ascites and carcinomatosis. Despite the extensive gastric involvement, the lack of gastrointestinal symptoms, such as obstruction or haemorrhage, was remarkable.

Anasarca may have been caused not only by HIV-related hypoalbuminemia but also by KS lymphatic dissemination and production of growth factors that increase vascular permeability ${ }^{[3]}$. We may also argue that the obstruction of lymphatic drainage may predispose to a proteinlosing enteropathy or protein malabsorption, and contribute directly to hypoalbuminemia and anasarca ${ }^{[3]}$.

The patient's clinical course was impressive and extremely fast. Some explanations for the fatal outcome can be discussed. Anasarca and immunodeficiency may predispose to septic shock and Gram-negative bacteremia by intestinal microbial translocation ${ }^{[2,3]}$. Fulminant MCD may also be considered as fever, multiple adenopathy, anasarca and hypoalbuminemia are frequent features but unfortunately, in this specific case, we were unable to exclude this entity ${ }^{[4]}$.

Recently, another KS- and HHV-8-associated condition has been described. This entity, called Kaposi's sarcoma human herpes virus inflammatory cytokine syndrome (KS HHV ICS), has some clinical manifestations resembling those of MCD, such as fever, wasting, cytopenias and hypoalbuminemia, but lymphadenopathy is not prominent and the histopathology of lymph nodes is not typical ${ }^{[4]}$. KS HHV ICS may provide another possible explanation for the systemic inflammatory response syndrome observed in our patient.

We also believe that patient's marked debility, opportunistic infection with HHV-8, as documented by active replication in blood and possible explosion of neoplastic disease, contributed to the fatal outcome.

In the era of highly efficient antiretroviral therapy, new chemotherapy and immunotherapy strategies for KS, effectiveness and safety of aggressive treatment in patients with advanced AIDS and disseminated KS remains uncertain ${ }^{[5]}$. This case should alert clinicians that opportunistic infections and terminal AIDS still occurs and it is important for clinicians to consider the possibility of HIV infection in every patient with an unexplained general deterioration. Also, the possibility of disseminated KS must be considered, even when the skin seems to be the only affected organ.

\section{REFERENCES}

1. Bhaijee F, Subramony C, Tang S, Pepper D. Human immunodeficiency virus-associated gastrointestinal disease: common endoscopic biopsy diagnoses. Patholog Res Int. 2011; 2011:1-8.

2. Ioachim H, Adsay V, Giancotti F, Dorsett B, Melamed J. Kaposi's sarcoma of internal organs. A multiparameter study of 86 cases. Cancer 1995;75:1376-1385.

3. Toschi E, Barillari G, Sgadari C, Palladino C, Cristofaro A, Ciccolella C et al. The HIV-1 Tat protein cooperates with basic fibroblast growth factor in inducing vascular permeability and edema: implications for AIDS-associated Kaposi's sarcoma (KS) pathogenesis. J Acquir Immune Defic Syndr 1999;21:A19.

4. Uldrick T, Wang V, O'Mahony D, Aleman K, Wyvill K, Marshall V et al. an interleukin-6-related systemic inflammatory syndrome in patients coinfected with Kaposi sarcomaassociated herpesvirus and HIV but without multicentric Castleman disease. Clin Infect Dis 2010;51:350-358.

5. Krown S. Highly active antiretroviral therapy in AIDS-associated Kaposi's sarcoma: implications for the design of therapeutic trials in patients with advanced, symptomatic Kaposi's sarcoma. J Clin Oncol 2003;22:399-402. 